\title{
New Explicit and Exact Traveling Waves Solutions To The Modified Complex Ginzburg Landau Equation
}

\section{Bienvenue Depelair}

University of Maroua: Universite de Maroua

\section{Alphonse Houwe}

University of Maroua: Universite de Maroua

\section{Hadi Rezazadeh}

Amol University of Special Modern Technologies

Ahmet Bekir ( $\sim$ bekirahmet@gmail.com )

Eskisehir https://orcid.org/0000-0001-9394-4681

\section{Mama Nsangou}

University of Maroua: Universite de Maroua

\section{Gambo Betchewe}

University of Maroua: Universite de Maroua

\section{Research Article}

Keywords: Exact solutions, function transformation method, modified Complex Ginzburg Landau equation

Posted Date: December 14th, 2021

DOI: https://doi.org/10.21203/rs.3.rs-1134759/v1

License: (c) (i) This work is licensed under a Creative Commons Attribution 4.0 International License. Read Full License 


\title{
New explicit and exact traveling waves solutions to the modified complex Ginzburg Landau equation
}

\author{
Depelair Bienvenue ${ }^{1}$, Alphonse Houwe $^{1}$, Hadi Rezazadeh $^{2}$, Ahmet Bekir $^{3 *}$, Mama Nsangou $^{1}$, Gambo Betchewe $^{4}$ \\ ${ }^{1}$ Department of Physics, Faculty of Science, the University of Maroua, P.O Box 814, Maroua, Cameroon. \\ ${ }^{2}$ Faculty of Engineering Technology, Amol University of Special Modern Technologies, Amol, Iran. \\ ${ }^{3}$ Neighbourhood of Akcaglan, Imarli Street, Number: 28/4, 26030, Eskisehir, Turkey. \\ ${ }^{4}$ Department of Physics, Faculty of Science, the University of Maroua, P.O Box 814, Maroua, Cameroon.
}

\begin{abstract}
This paper applies function transformation method to obtain under certain conditions bright, dark, kink and W-shaped dark solitons waves solutions to the modified Complex Ginzburg Landau Equation (CGLE). These new obtained solutions can be useful in many applications such as communication, medicine, hydrodynamic, thermodynamic just to name a few and can allow to explain physical phenomena.
\end{abstract}

Keywords: Exact solutions; function transformation method ; modified Complex Ginzburg Landau equation.

\section{Introduction}

Solitary waves propagation in nonlinear media received great attention these recent years because of their extensive applications in the field of ultra-fast science [1-7]. The latter were known through the pioneers works refs. $[8,9]$, like wave that can propagate over a long distance without undergoing modification and also maintains its origins. However, in various works solitary waves terms and solitons are generally related in the definition through properties such as soliton is a solitary waves having permanent form, localized, interact highly with other solitons and can retain its oneness.

More precisely, the important remark which has pointed out the interest of solitary waves theory in many fields is that the nonlinear and dispersion terms of nonlinear evolution equations can balance to form solitons solutions. Thus, there is a category of solitary waves in literature namely bell-shape, periodic solutions, kinks wave, peaked solitary waves, solitons exhibited cups and so on. Furthermore, the most commonly used names are the bright, dark and singular solitons $[4-9,11]$.

Several studies are made by researchers in order to exhibit how much solitons and other fractional solutions of nonlinear evolution equations are important to bring efficient solution to the problem of communication, medicine, hydrodynamic by avoiding signal loose $[1-7,11,12]$. So, in the case of nonlinear optical fibers, optical amplifiers have to be employed to compensate this loss. For example, soliton propagating in a monomode optical fibers have been used for future bits in long move communications and other fiber optic

${ }^{*}$ Corresponding author: E-mail: bekirahmet@gmail.com 
founded ultra fast pulse inspection devices. Indeed, high bit rate data relocation over a distance of thousands kilometers using optical solitons and smash erbium doped fiber amplifiers appeared to be technologically viable in close prospectives. In addition, it has been used a periodic fluctuation of the core diameter to master soliton parameters. Otherwise, it has been demonstrated that solitons can propagate over 14,000 $\mathrm{km}$ using an amplifying signals technique in an optical fiber. In addition, the use of the soliton in the field of chemistry has ensured the electrical conduction of conductive plastics, while acoustic soliton have contributed to minimize waves shock when trains entered tunnels. More recently, it has been emphasized the behavior of the new soliton solution to the longitudinal wave equation in a magneto-electro-elastic circular rod by applying the extended trial equation method. We also noted that solitons appeared on the surface during nonlinear distortions in DNA functions, where nonlinear interactions can give rise to stable excitations. Stable excitation (solitons) play an important role in the key processes of respiration, replication and transcription of DNA [1-7,11-41,43-51].

Among these studies of soliton-like solutions which propagated by conserving its velocity and shaped, several studies have been conducted on the modified Complex Ginzburg Landau Equation (CGLE) [15-18], [22].

CGLE is a complex nonlinear equation which depicts generically the dynamics of wavering, spatially prolonged systems close to the start of oscillations. This equation describes also a variety of physical phenomena in plasmas, optical wave guides and fibers, Bose Einstein condensation, phase transitions, bimolecule dynamics, open flow motions, spatially extended non equilibrium systems [15-18]. We cite some examples such as Osman et al. who study Complex wave structures for abundant solutions related to the CGLE model [22], in this work, some wave structures as solutions to the CGL equation have been reported. Mohamadou et al. have conducted study on Pattern selection and modulation instability in one dimensional modified CGL equation [18], they have highlighted that modulation unstable pattern is selected and propagated into an initially unstable motionless state in the system.

In the present work, we will investigate some solitons solutions such as bright, dark, kink and W-shaped dark, and other periodic structures for the modified Complex Ginzburg Landau equation. To reach this aim, the present paper is structured as follows: in section (2), we exhibit the model; in section (3), solutions are given to the model by applying function transformation method; in section (4), we summarize the work by giving some important remarks and graphical representations.

\section{Skeleton of the model}

The complex field $\psi(x, t)$ of 1-D modified CGL equation is represented as follows $[15,18,22]$

$$
i \psi_{t}+\alpha \psi_{x x}+\lambda|\psi|^{2} \psi=c \frac{\psi_{x} \psi_{x}^{*}}{\psi^{*}}+d \nabla^{2}\left(\sqrt{\psi \psi^{*}}\right) \sqrt{\psi / \psi^{*}}+i \gamma \psi,
$$

where the operator $\nabla^{2} \equiv \frac{\partial^{2}}{\partial x^{2}}$ and $\alpha, \lambda, c, d$ and $\gamma$ are reals.

Meanwhile, the expression $d \nabla^{2}\left(\sqrt{\psi \psi^{*}}\right) \sqrt{\psi / \psi^{*}}$ in the right side of equation (1) can be rewritten as 


$$
d \nabla^{2}\left(\sqrt{\psi \psi^{*}}\right) \sqrt{\psi / \psi^{*}}=d\left[\frac{1}{2} \frac{\partial^{2}\left(\psi \psi^{*}\right)}{\partial x^{2}} \psi \psi^{*}-\frac{1}{4}\left(\frac{\partial\left(\psi \psi^{*}\right)}{\partial x}\right)^{2}\right] \frac{1}{\psi \psi^{* 2}} .
$$

Employing Eq.(2) into Eq.(1), the CGL equation can be explicitly rewritten as follows

$$
i \psi_{t}+\alpha \psi_{x x}+\lambda|\psi|^{2} \psi=c \frac{\psi_{x} \psi_{x}^{*}}{\psi^{*}}+d\left[\frac{1}{2} \frac{\partial^{2}\left(\psi \psi^{*}\right)}{\partial x^{2}} \psi \psi^{*}-\frac{1}{4}\left(\frac{\partial\left(\psi \psi^{*}\right)}{\partial x}\right)^{2}\right] \frac{1}{\psi \psi^{*}} .+i \gamma \psi
$$

Next, we employ the transformation hypothesis to build solitary waves to the CGL equation and discussed the obtained miscellaneous soliton solutions. So, we set

$$
\psi(x, t)=\Psi(\xi) \exp [i \theta(x, t)], \quad \theta(x, t)=\kappa x-\omega t+\theta_{0}, \quad \xi=x-v t,
$$

Inserting Eq.(4) into Eq.(1) gives

$$
(\alpha-d) \Psi_{\xi \xi}-\frac{c \Psi_{\xi}^{2}}{\Psi}+\lambda \Psi^{3}+\left(\omega-\alpha k^{2}-c k^{2}\right) \Psi=0,
$$

and

$$
(2 \alpha k-v) \Psi_{\xi}-\gamma \Psi=0 .
$$

The latter denotes real and imaginary parts respectively. From Eq.(6), we obtain

$$
\Psi_{\xi}=\frac{\gamma \Psi}{2 \alpha k-v} .
$$

Inserting Equation Eq.(7) into equation Eq.(5) yields to

$$
\Psi_{\xi \xi}+\frac{\lambda}{\alpha-d} \Psi^{3}+\frac{1}{\alpha-d}\left(\omega-\alpha k^{2}-c k^{2}-\frac{c \gamma^{2}}{(2 \alpha k-v)^{2}}\right) \Psi=0 .
$$

The integration Eq.(8) with respect to $\xi$ gives the following expression

$$
\Psi_{\xi}^{2}=\frac{\lambda}{2(d-\alpha)} \Psi^{4}+\frac{1}{d-\alpha}\left(\omega-\alpha k^{2}-c k^{2}-\frac{c \gamma^{2}}{(2 \alpha k-v)^{2}}\right) \Psi^{2}+K
$$

where $K$ is an integration constant. After setting the following constants as $c_{1}=\frac{1}{d-\alpha}\left(\omega-\alpha k^{2}-c k^{2}-\frac{c \gamma^{2}}{(2 \alpha k-v)^{2}}\right)$, and $c_{2}=\frac{\lambda}{2(d-\alpha)}$, Eq.(9) takes the following expression

$$
\Psi_{\xi}^{2}=c_{2} \Psi^{4}+c_{1} \Psi^{2}+K
$$

It is important to emphasize that nonlinear ordinary equation (NODE) represents by Eq.(8) which depends only on $\xi$, can be solved by employing auxiliary equation methods, rational function method or ansatz methods. But to point out analytical solutions in this work, we apply the function transformation method [52]. Recently, in ref. [10], the two-dimensional differential transform method have been employed to facilitate computational difficulties. The used of this method usually imposed the boundary condition like the decomposition method. The important matter is that this method facilitated the investigation of the solitary waves without computer codes.

Employing mathematical transformation Eq.(10) takes the form of

$$
\frac{d \Psi}{\sqrt{\frac{1}{d-\alpha}\left(\omega-\alpha k^{2}-c k^{2}-\frac{c \gamma^{2}}{(2 \alpha k-v)^{2}}\right) \Psi^{2}+\frac{\lambda}{2(d-\alpha)} \Psi^{4}+K}}=d \xi .
$$




\section{$3 \quad$ Exact soliton solutions}

According to the different values of constant of integration $K$ and employing the same transformation in ref. [52], equation Eq.(11) has different explicit and exact analytic solutions as follows

case(I) For localized solutions, we will consider $K=0$ and $\frac{\lambda}{2(d-\alpha)}>0$ in Eq.(11). Therewith, the following solitary wave solution for the ordinary differential Eq.(1) can be obtained as

$$
\Psi(\xi)=\frac{4 \sqrt{\Upsilon} \exp \left(\sqrt{\Upsilon}\left(\xi+\xi_{0}\right)\right)}{4 \Gamma \exp \left(2 \sqrt{\Upsilon}\left(\xi+\xi_{0}\right)\right)-1}
$$

and

$$
\Psi(\xi)=\frac{4 \sqrt{\Upsilon} \exp \left(\sqrt{\Upsilon}\left(\xi+\xi_{0}\right)\right)}{4 \Gamma \exp \left(2 \sqrt{\Upsilon} \xi_{0}\right)-\exp (2 \sqrt{\Upsilon} \xi)}
$$

With $\xi_{0}$ a second constant of integration. However, $\Upsilon=\sqrt{\frac{1}{d-\alpha}\left(\omega-\alpha k^{2}-c k^{2}-\frac{c \gamma^{2}}{(2 \alpha k-v)^{2}}\right)}$ and $\Gamma=\frac{\lambda}{2(-d+\alpha)}$. Then, the solution of the modified CGL equation Eq.(1) can stay in the following form

$$
\psi_{1,1}(x, t)=\frac{4 \sqrt{\Upsilon} \exp \left(\sqrt{\Upsilon}\left(x-v t+\xi_{0}\right)\right)}{4 \Gamma \exp \left(2 \sqrt{\Upsilon}\left(x-v t+\xi_{0}\right)\right)-1} \exp \left(i\left(\kappa x-\omega t+\theta_{0}\right)\right)
$$

and

$$
\psi_{1,2}(x, t)=\frac{4 \sqrt{\Upsilon} \exp \left(\sqrt{\Upsilon}\left(x-v t+\xi_{0}\right)\right)}{4 \Gamma \exp \left(2 \sqrt{\Upsilon} \xi_{0}\right)-\exp (2 \sqrt{\Upsilon}(x-v t))} \exp \left(i\left(\kappa x-\omega t+\theta_{0}\right)\right)
$$

The constraint relation is $\frac{1}{d-\alpha}\left(\omega-\alpha k^{2}-c k^{2}-\frac{c \gamma^{2}}{(2 \alpha k-v)^{2}}\right)>0$. The validity of Eq.(14) arises on $4 \Gamma \exp (2 \sqrt{\Upsilon}(x-$ $\left.\left.v t+\xi_{0}\right)\right)-1 \neq 0$ and the same time the validity of Eq.(15) is given by $4 \Gamma \exp \left(2 \sqrt{\Upsilon} \xi_{0}\right)-\exp (2 \sqrt{\Upsilon}(x-v t)) \neq 0$.

\section{$\operatorname{Case}(\mathbf{I I})$}

(a) If $K=0, \xi_{0}=0, \frac{1}{d-\alpha}\left(\omega-\alpha k^{2}-c k^{2}-\frac{c \gamma^{2}}{(2 \alpha k-v)^{2}}\right)>0$ and $\frac{\lambda}{2(d-\alpha)}<0$, the following bell-shaped solitary wave solution of equation Eq.(10) can be obtained

$$
\Psi(\xi)=\frac{\Upsilon}{\Gamma} \operatorname{sech}(\sqrt{\Upsilon} \xi)
$$

Hence, the first bright soliton solutions a of Eq. (1) is

$$
\psi_{2,1}(x, t)=\frac{\Upsilon}{\Gamma} \operatorname{sech}(\sqrt{\Upsilon}(x-v t)) \exp \left(i\left(\kappa x-\omega t+\theta_{0}\right)\right) .
$$

(b) If $K=0, \xi_{0}=0, \frac{1}{d-\alpha}\left(\omega-\alpha k^{2}-c k^{2}-\frac{c \gamma^{2}}{(2 \alpha k-v)^{2}}\right)<0$ and $\frac{\lambda}{2(d-\alpha)}<0$ in equation Eq.(11), the following triangular solution of equation Eq.(10) can be obtained

$$
\Psi(\xi)=\frac{-\Upsilon}{\Gamma} \operatorname{sech}(\sqrt{-\Upsilon} \xi)
$$

Then, equation (1) has the following solution

$$
\psi_{2,2}(x, t)=\frac{-\Upsilon}{\Gamma} \operatorname{sech}(\sqrt{-\Upsilon}(x-v t)) \exp \left(i\left(\kappa x-\omega t+\theta_{0}\right)\right) .
$$

\section{Case(III)}

(a) If $K=\frac{-4}{\lambda(d-\alpha)}\left(\omega-\alpha k^{2}-c k^{2}-\frac{c \gamma^{2}}{(2 \alpha k-v)^{2}}\right)^{2}, \xi_{0}=0$, while $\frac{1}{d-\alpha}\left(\omega-\alpha k^{2}-c k^{2}-\frac{c \gamma^{2}}{(2 \alpha k-v)^{2}}\right)<0$ and $\frac{\lambda}{2(d-\alpha)}<0$, the following kink-shaped solitary wave solution of Eq.(10) can be found 


$$
\Psi(\xi)=\frac{-\Upsilon}{\Gamma} \tanh (\sqrt{-\Upsilon} \xi) .
$$

Then, equation (1) has the following solution

$$
\psi_{3,1}(x, t)=\frac{-\Upsilon}{\Gamma} \tanh (\sqrt{-\Upsilon}(x-v t)) \exp \left(i\left(\kappa x-\omega t+\theta_{0}\right)\right) .
$$

However, the constraint relation of the obtained Eq.(19) is $\Upsilon<0$ and the constraint relation of Eq.(21) is $\Upsilon>0$.

(b) If $K=\frac{-4}{\lambda(d-\alpha)}\left(\omega-\alpha k^{2}-c k^{2}-\frac{c \gamma^{2}}{(2 \alpha k-v)^{2}}\right)^{2}$ and $\xi_{0}=0$, while $\frac{1}{d-\alpha}\left(\omega-\alpha k^{2}-c k^{2}-\frac{c \gamma^{2}}{(2 \alpha k-v)^{2}}\right)>0$ and $\frac{\lambda}{2(d-\alpha)}<0$, the following triangular solution of equation Eq.(10) is unearthed

$$
\Psi(\xi)=\frac{\Upsilon}{\Gamma} \tan (\sqrt{\Upsilon} \xi)
$$

Then, the solution of equation Eq.(1) becomes

$$
\psi_{3,2}(x, t)=\frac{\Upsilon}{\Gamma} \tan (\sqrt{\Upsilon}(x-v t)) \exp \left(i\left(\kappa x-\omega t+\theta_{0}\right)\right) .
$$

Case(IV) Equation (6) admits three jacobian elliptic function solutions as follows

(a) If $K=\frac{\Upsilon^{2} m^{2}\left(m^{2}-1\right)}{\Gamma\left(2 m^{2}-1\right)^{2}}$ with $\frac{1}{d-\alpha}\left(\omega-\alpha k^{2}-c k^{2}-\frac{c \gamma^{2}}{(2 \alpha k-v)^{2}}\right)>0$. While $\frac{\lambda}{2(d-\alpha)}>0$, the following solution for equation Eq.(10) can be obtained

$$
\Psi(\xi)=\sqrt{\frac{-\Upsilon m^{2}}{\Gamma\left(2 m^{2}-1\right)}} \operatorname{cn}\left(\sqrt{\frac{\Upsilon}{2 m^{2}-1}} \xi\right) .
$$

Then, equation Eq.(1) has the following solution

$$
\psi_{4,1}(x, t)=\sqrt{\frac{-\Upsilon m^{2}}{\Gamma\left(2 m^{2}-1\right)}} c n\left(\sqrt{\frac{\Upsilon}{2 m^{2}-1}}(x-v t)\right) \exp \left(i\left(\kappa x-\omega t+\theta_{0}\right)\right) .
$$

(b) If $K=\frac{\Upsilon^{2}\left(1-m^{2}\right)}{\Gamma\left(2-m^{2}\right)^{2}}, \frac{1}{d-\alpha}\left(\omega-\alpha k^{2}-c k^{2}-\frac{c \gamma^{2}}{(2 \alpha k-v)^{2}}\right)>0$ and $\frac{\lambda}{2(d-\alpha)}>0$, the following solution for equation Eq.(10) can be found

$$
\Psi(\xi)=\sqrt{\frac{-\Upsilon}{\Gamma\left(2-m^{2}\right)}} d n\left(\sqrt{\frac{\Upsilon}{2-m^{2}}} \xi\right) .
$$

Thereafter, Eq.(1) has the following solution

$$
\psi_{4,2}(x, t)=\sqrt{\frac{-\Upsilon}{\Gamma\left(2-m^{2}\right)}} d n\left(\sqrt{\frac{\Upsilon}{2-m^{2}}}(x-v t)\right) \exp \left(i\left(\kappa x-\omega t+\theta_{0}\right)\right) .
$$

(c) If $K=\frac{\Upsilon^{2} m^{2}}{\Gamma\left(m^{2}+1\right)^{2}}, \frac{1}{d-\alpha}\left(\omega-\alpha k^{2}-c k^{2}-\frac{c \gamma^{2}}{(2 \alpha k-v)^{2}}\right)<0$ and $\frac{\lambda}{2(d-\alpha)}<0$, the following solution for Eq.(10) gives

$$
\Psi(\xi)=\sqrt{\frac{-\Upsilon m^{2}}{\Gamma\left(m^{2}+1\right)}} \operatorname{sn}\left(\sqrt{\frac{-\Upsilon}{m^{2}+1}} \xi\right) .
$$



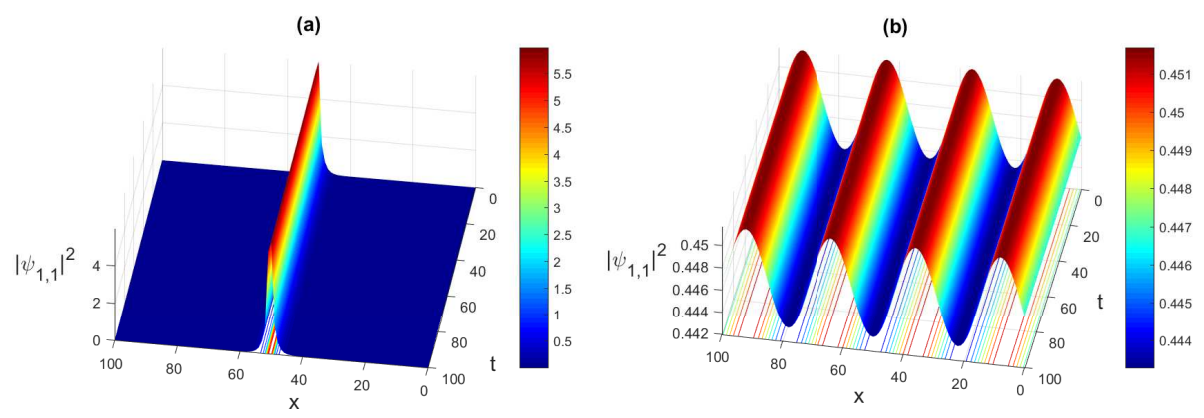

Figure 1: The plot of the bright soliton of $\left|\psi_{1,1}\right|^{2}$ of the solution Eq.(14) (a) for $d=1.000012, \alpha=$ $1, k=0.000125, c=0.00001045, \lambda=-0.047, \xi_{0}=0.0012, v=0.000015$ and (b) $d=.000012, \alpha=1, k=$ $0.000125, c=1.01045, \lambda=-0.047, \xi_{0}=0.0012, v=0.000015$ while $-150 \leq x \leq 150$ respectively.

Then, Eq.(1) turns to

$$
\psi_{4,3}(x, t)=\sqrt{\frac{-\Upsilon m^{2}}{\Gamma\left(m^{2}+1\right)}} \operatorname{sn}\left(\sqrt{\frac{-\Upsilon}{m^{2}+1}}(x-v t)\right) \exp \left(i\left(\kappa x-\omega t+\theta_{0}\right)\right) .
$$

The following restrictive condition in the above solutions is $\frac{1}{d-\alpha}\left(\omega-\alpha k^{2}-c k^{2}-\frac{c \gamma^{2}}{(2 \alpha k-v)^{2}}\right)>0$. In the same time Eq.(25) is invalid for $m= \pm \frac{\sqrt{2}}{2}$ and Eq.(27) is valid for $m \neq \pm \sqrt{2}$ along with $\Gamma>0$.

The obtained above results of the 1D-complex Ginzburg Landau are recovered by using the direct integration (function transformation). These solutions are rational function solutions, bright soliton, dark soliton and jacobian elliptic function solutions. Compared to refs. [18-20], it is unearthed some new results (i.e. Eq.(14), Eq.(15), Eq.(17) and Eq.(19)). Beside, using an adequate parameters of the model, some additional results are obtained such as dark and an additional jacobian elliptic function solutions compared to $[19,20]$. These results are very important in diverse field such as optic fibers, ocean engineering, fluid mechanics, biology and so on [20-23]. Between others, it should be emphasized that, without using computer codes, the obtained results are comparatively look the same with those investigated by adopting several integration technics in refs. [25-33] just to list a few.

Furthermore, by considering zero value the integration constant, we yield to Eq.(14), Eq.(15) which are rational solutions. At the same time, to set the integration constant to zero $(K=0)$ and taking $\left(\xi_{0}=0\right)$, it is gained bright solitons. Moreover, taking now the value of integration constant different to zero and 

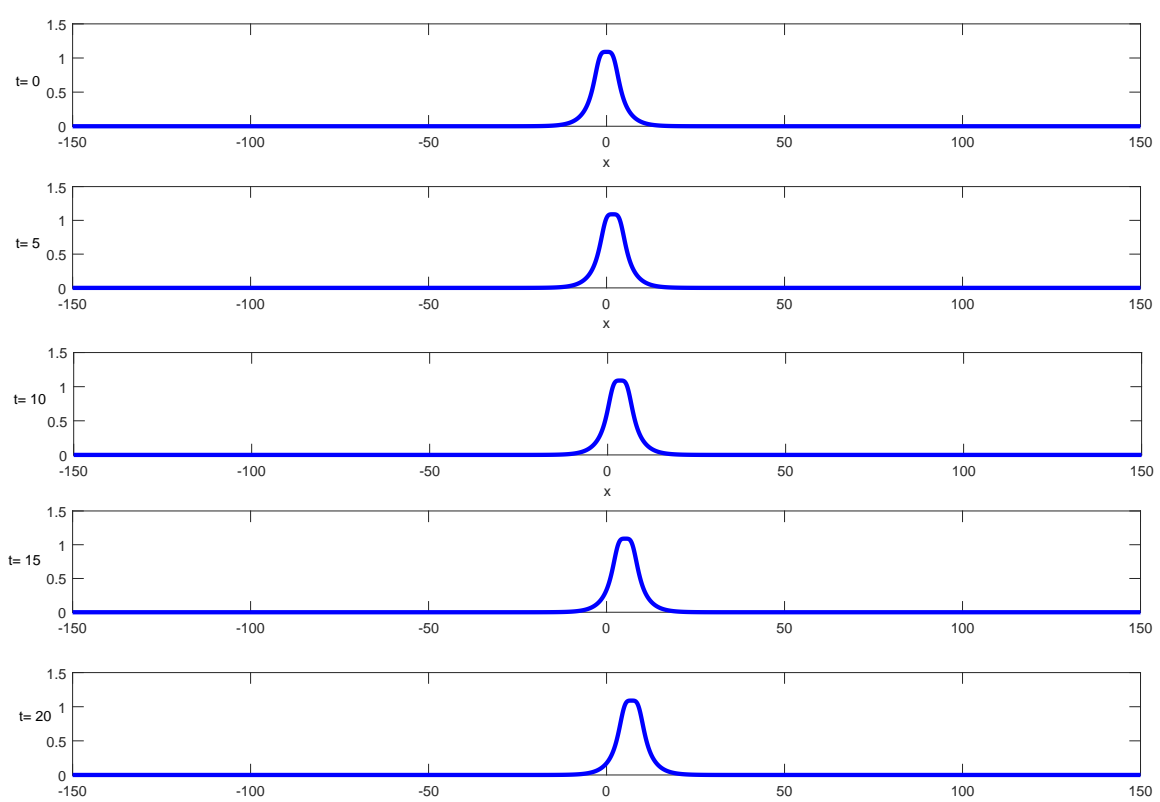

Figure 2: The plot of the bright soliton of $\left|\psi_{2,1}\right|^{2}$ of the solution Eq.(17) for $d=0.02, \alpha=1, k=0.000125, c=$ $10.045, \lambda=0.47, v=0.25$ at $t=0, t=5, t=10, t=15, t=20$ and $-150 \leq x \leq 150$ respectively.
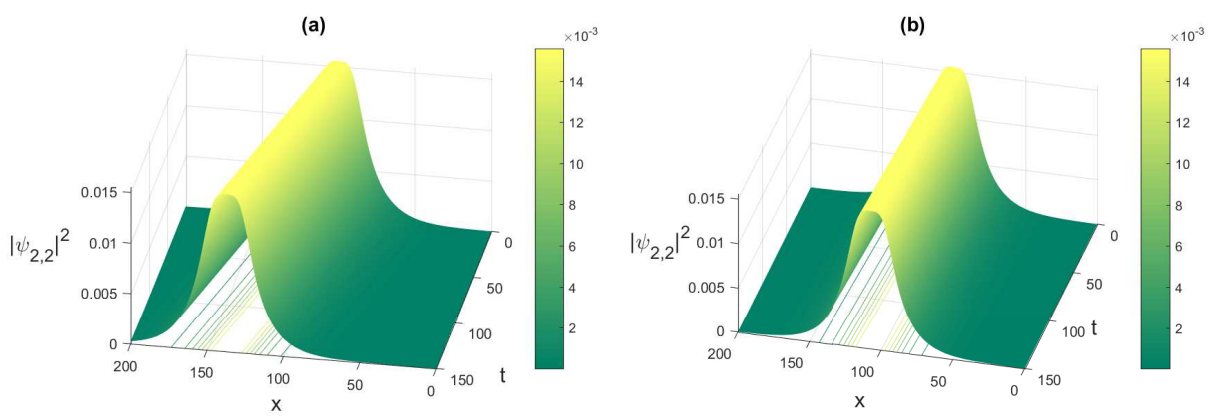

Figure 3: Spatiotemporal plot evolution of bright soliton $\left|\psi_{2,2}\right|^{2}$ of the solution Eq.(19) for $d=0.02, \alpha=$ $1, \lambda=0.047, k=0.000125, c=0.04 a t(a) v=0.55,(b) v=0.35$ respectively. 
(a)

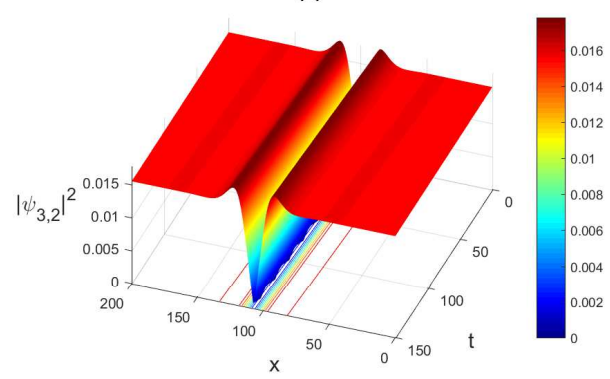

(b)

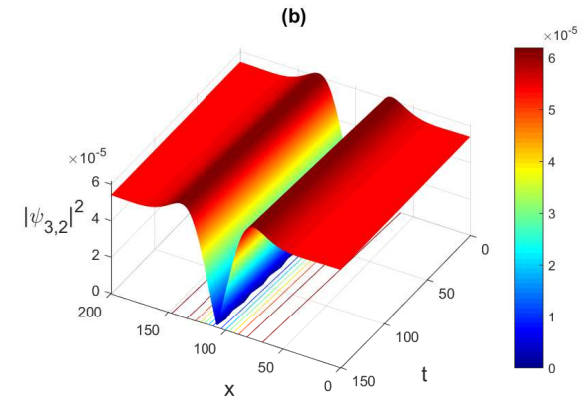

Figure 4: Spatiotemporal plot evolution of W-shape dark $\left|\psi_{3,2}\right|^{2}$ of the solution Eq.(23) for (a) $d=0.2, \alpha=$ $1, c=0.00104, k=0.00125, \gamma=0.02, v=0.34, \lambda=100.47$, and (b) $d=0.2, \alpha=1, c=0.00104, k=$ $0.00125, \gamma=0.02, v=0.75, \lambda=100.47$ respectively.

assuming $\left(\xi_{0}=0\right)$, we unearth dark soliton. The constraint relation on the dark soliton reads $\frac{1}{d-\alpha}\left(\omega-\alpha k^{2}-\right.$ $\left.c k^{2}-\frac{c \gamma^{2}}{(2 \alpha k-v)^{2}}\right)<0$. While the jacobian elliptic function solutions are given under the constraint relation $\frac{1}{d-\alpha}\left(\omega-\alpha k^{2}-c k^{2}-\frac{c \gamma^{2}}{(2 \alpha k-v)^{2}}\right)>0$ and $\frac{\lambda}{2(d-\alpha)}>0$. The obtained self-localized waves of the CGLE can describe the dynamic of the solitary waves in various field of science and engineering.

Figure 1 depicts the spatiotemporal evolution of $\left|\psi_{1,1}\right|^{2}$ of Eq.(14), and Figure 2 is the bright soliton of $\left|\psi_{2,1}\right|^{2}$. However, Figure 3 shows the spatiotemporal evolution of the bright soliton $\left|\psi_{2,2}\right|^{2}$ of Eq.(18) at different values of the soliton speed. Furthermore, in figure 4 the W-shaped dark (a) and the corresponding dark soliton (b) of $\left|\psi_{3,2}\right|^{2}$ of Eq.(23) are stressed. The obtained bright and dark solitons solutions can be useful in communication system via the optical fibers [34-41]. These results will certainly encourage the search for solutions to nonlinear differential equations which seem to be difficult to handle without computer codes. However, the obtained Figures in this work were depicted with help of MATLAB software.

\section{Conclusions}

In this paper, we investigate exact solutions to the modified Complex Ginzburg Landau equation. The first step consists to use the transformation hypothesis to obtain the nonlinear ordinary equation (NODE). To unearth exact traveling waves solutions to the NODE, we employ the function transformation method which does not use mathematics codes. From there, considering certain conditions on the CGL equation 
parameters, it is revealed bright, dark and rational solutions. These results have been illustrated over the 3-D and 2-D graphical representation by the help of MATLAB software. It is also important to emphasize that the obtained NODE could be handle by other methods. More recently, the CGL model has been investigated in refs. [16-18,22]. They have unearthed pulses, front, kink soliton and periodic solutions by adopting Painleve analysis and Hirota bilinear $[17,17]$. The steady state of these results have been also set

out in ref. [17]. In this paper, the obtained results are new compared to [15-18,23-31] and will certainly have an important effects in many applications in the field of solitary waves such as communication, medicine, hydrodynamic, thermodynamic and will extensively be helpful to explain some phenomena of ultra fast science. Next, others methods will be applied in order to emphasize and show out relevant interpretations.

\section{$5 \quad$ Availability of supporting data}

Not applicable.

\section{Competing interests}

The authors declare that they have no competing interests.

\section{References}

[1] S Konar, A Biswas Journal of Electromagnetic Waves and Applications 191443 (2005)

[2] M Arshad, A R Seadawy, D Lu Journal of Electromagnetic Waves and Applications 311711 (2017)

[3] S Konar, J Kumar, P K J Sen Nonlinear Optical Physics 8 Materials 8492 (1999)

[4] S Crutcher, A Biswas, M D Aggrawal, M E Edwards Journal of Electromagnetic Waves and Applications $20761(2006)$

[5] P G Kevrekidis, D J Frantzeskakis Reviews in Physics 1140 (2016)

[6] B Mandal, A R Chowdhury Journal of Electromagnetic Waves and Applications 21123 (2007)

[7] A Biswas Quantum Phys. Lett. 179 (2012)

[8] P G Drazin, R S Johnson Solitons : an introduction Cambridge University Press, Cambridge (1996)

[9] W Hereman Shallow Water Waves and Solitary Waves Springer-Verlag, Heibelberg (2009)

[10] F Ayaz Applied Mathematics and Computation 143361 (2003)

[11] B A Malomed, I M Skinner, R S Tasgal Optics communications 139247 (1997)

[12] M S Osman, H Rezazadeh, M Eslami, A Neirameh, M Mirzazadeh Univ Politechnica Bucharest Sci. Bull-Ser A-Appl Math Phys. 80267 (2018) 
[13] R J Hirota J. Math Phys. 14 (1973) 805.

[14] H Majid, A Mahrokh, M M O Mendéz Optik 202163695 (2020)

[15] H Rezazadeh Optik 167218 (2018)

[16] E Yomba, T C Kofane Chaos, Solitons and Fractals 15197 (2003)

[17] W P Hong Z. Naturforsch 62a 373 (2007)

[18] A Mohamadou, A K Jiotsa, T C Kofane Chaos, Solitons and Fractals 24957 (2005)

[19] S Shamseldeen, M S A Latif, A Hamed, H Nour Communication in Mathematical Modeling and Applications 239 (2017)

[20] J Zhang, C Dai Chinese Optics Letters 3295 (2005)

[21] H Alphonse, M B Hubert, N Savaissou, D Jerome, M Justin, G Betchewe, A Biswas, M Ekici Optik 179861 (2019)

[22] M S Osman, D Lu, M M A Khater, R A M Attia Optik 192162927 (2019)

[23] R Gangwar, S P Singh, N Singh Progress In Electromagnetics Research 74157 (2007)

[24] C Yan Physics Letters A 22477 (1996)

[25] A R Seadawy, M Jalil Results in Physics 81158 (2018)

[26] S S Ehab, A R Seadawy, Y Xiaohua The European Physical Journal Plus 131425 (2016)

[27] A R Seadawy, K El-Rashidy Results in Physics 81216 (2018)

[28] A R Seadawy, S Z Alamri Results in Physics 8286 (2018)

[29] Abdullah, A R Seadawy, J Wang Results in Physics 74269 (2017)

[30] A R Seadawy European Physical Journal Plus 132518 (2017)

[31] A R Seadawy, K El-Rashidy Pramana-Journal of Physics 8720 (2016)

[32] A R Seadawy Pramana-Journal of Physics 8911 (2017)

[33] A Asghar, A R Seadawy, D Lu Optik 144475 (2017)

[34] N Savaissou, S Abbagari, H Alphonse, M Inc, B Gambo, S Y Doka Commun. Theor. Phys. 72065501 (2020)

[35] N Savaissou, H Alphonse, H Rezazadeh, A Bekir, B Gambo, S Y Doka Modern Physics Letters B 32 $2050246(2020)$

[36] N Savaissou, H Alphonse, B Gambo, M Inc, S Y Doka Physica Scripta 95085108 (2020) 
[37] N Savaissou, B Gambo, H Rezazadeh, A Bekir, S Y Doka Optical and Quantum and Electronics 52318 (2020)

[38] A Souleymanou, A Korkmaz, H Rezazadeh, S P Takougoum Mukam, A Bekir Modern Physics Letters B 312050038 (2019)

[39] A Souleymanou, K K Ali, H Rezazadeh, M Eslami, M Mirzazadeh, A Korkmaz Pramana-J. Phys. 93 $27(2019)$

[40] A Souleymanou, V K Kuetche, B T Bouetou, T C Kofane Chin. Phys. Lett. 28120501 (2011)

[41] A Souleymanou, V K Kuetche, B T Bouetou, T C Kofane Commun. Theor. Phys. 5710 (2012)

[42] I Mustafa, Y Abdullahi, T A Aliyu, B Dumitru Optik 16265 (2018)

[43] I Mustafa, Y Abdullahi, T A Aliyu, B Dumitru Optical and Quantum Electronics 50139 (2018)

[44] I A Aliyu, T Fairouz, I Mustafa, Y Abdullahi, B Dumitru Journal of Modern Optics 66136 (2019)

[45] T A Aliyu, I Mustafa, Y Abdullahi, B Dumitru Optik 178923 (2019)

[46] Y Abdullahi, I Mustafa, B Dumitru Frontiers in Physics 734 (2019)

[47] A A Omar J. Appl. Math. Comput. 59227 (2019)

[48] A A Omar, R Hasan Neural Comput and Applic. 302595 (2018)

[49] A A Omar Fundamenta Informaticae 166111 (2019)

[50] A A Omar Calcolo 5531 (2018)

[51] A A Omar, S Nabil Journal of Porous Media 411 (2019)

[52] A R Seadawy Applied Mathematics Letters 25687 (2012) 